\title{
ENERGY OPTIMISATION SCHEMES FOR WIRELESS SENSOR NETWORK
}

\author{
Vivekanand Jha, Elisha Chauhan,Harshpreet Kaur and Neeharika \\ Taneja
}

Department of Computer Science, Indira Gandhi Institute of technology GGSIPU, Delhi, India

\begin{abstract}
A sensor network is composed of a large number of sensor nodes, which are densely deployed either inside the phenomenon or very close to it. Sensor nodes have sensing, processing and transmitting capability. They however have limited energy and measures need to be taken to make op-timum usage of their energy and save them from task of only receiving and transmitting data without processing. Various techniques for energy utilization optimisation have been proposed Ma jor players are however clustering and relay node placement. In the research related to relay node placement, it has been proposed to deploy some relay nodes such that the sensors can transmit the sensed data to a nearby relay node, which in turn delivers the data to the base stations. In general, the relay node placement problems aim to meet certain connectivity and/or survivabil-ity requirements of the network by deploying a minimum number of relay nodes. The other approach is grouping sensor nodes into clusters with each cluster having a cluster head $(\mathrm{CH})$. The $\mathrm{CH}$ nodes aggregate the data and transmit them to the base station (BS). These two approaches has been widely adopted by the research community to satisfy the scala-bility objective and generally achieve high energy efficiency and prolong network lifetime in large-scale WSN environments and hence are discussed here along with single hop and multi hop characteristic of sensor node.
\end{abstract}

\section{Keywords}

Clustering,Dominating Set,Relay Node,Sensor Node and Sink

\section{INTRODUCTION}

A wireless sensor network is composed of hundreds sensor nodes which use wireless links to perform distributed sensing tasks. Each sensor node includes a sensing module, a computing module, memory and a wireless communication module with a very limited communication range. Wireless sensor network has received intensive research attentions due to its enormous application potential in battlefield surveillance, environmental monitoring, biomedical

David C. Wyld, et al. (Eds): CCSEA, SEA, CLOUD, DKMP, CS \& IT 05, pp. 103-114, 2012.

(C) CS \& IT-CSCP 2012

DOI : $10.5121 /$ csit.2012.2212 
observation and other fields. The three basic requirements for designing efficient wireless sensor networks are scalability, fault-tolerance and energy efficiency. A sensor network, comprising of a number of sensor nodes, is usually required to cover a large geographic area. New sensor nodes may be added to the network and existing sensor nodes may be- come inoperative at any time. This large scale and frequently changing network requires scalable protocols and algorithms. Factors, such as energy depletion, harsh environmental conditions, and/or malicious attacks may result in node failures in a wireless sensor net- work. Therefore, survivability of sensor networks is a critical design goal. Moreover, energy is one of the most precious resource in wireless sensor networks. Sensor nodes are normally powered by batteries and can only last for a fairly short period of time if operated at high transmission power levels.[1]

These sensor nodes sense and communicate data over multiple hops to the base stations. The following two energy-related issues have emerged: (i) sensor nodes will spend most of their energy on relaying data, which is not economical because they are relatively expensive; (ii) sensor nodes closer to the sink are required to relay more data and thus will have a considerably shorter lifetime, resulting in network partitioning and limitation of the network lifetime $[11,12]$.

The fact that the energy requirement for transmission is a super-linear function of the transmission distance[1] necessitates short range communication to improve network lifetime[6]. To prolong network lifetime while meeting certain network specifications, one proposed direction is to deploy a small number of relay nodes (RNs) in the WSN such that they can communicate with the SNs and other RNs [2], [3], [4], [5], [6], [7], [8], [9], [10]. Relay node placement problems can be classified into either single-tiered or two-tiered based on the routing structures [5], [6], [9], [14] and into either connected or survivable based on the connectivity requirements [13], [5], [7], [15]. In single-tiered relay node placement, a SN also forwards packets received from other nodes. In two-tiered relay node placement, a SN forwards its sensed information to a $\mathrm{RN}$ or a base station (BS), but does not forward packets received from other nodes. In connected relay node placement, we place a small number of RNs to ensure that each sensor node is connected with a base station through a bidirectional path. In survivable relay node placement, we place a small number of RNs to ensure that each sensor node is connected with two base stations (or the only base station, in case there is only one base station) through two node-disjoint bidirectional paths.

In order to satisfy the scalability objective and achieve high energy efficiency in largescale WSN environment, the other approach widely used is clustering. In clustering, sensors nodes are grouped into clusters. In clustering approaches, all the nodes are efficiently divided into disjoint subsets and within each such subset a cluster head is elected[26]. The BS is the data processing point for the data received from the sensor nodes, and where the data is accessed by the end user. It is generally considered fixed and at a far distance from the sensor nodes. The $\mathrm{CH}$ nodes actually act as gateways between the sensor nodes and the BS. The function of each $\mathrm{CH}$, as already mentioned, is to perform common functions for all the nodes in the cluster, like aggregating the data before sending it to the BS. In some way, the $\mathrm{CH}$ is the sink for the cluster nodes, and the BS is the sink for the CHs. Moreover, this structure formed between the sensor nodes, the sink $(\mathrm{CH})$, and the BS can be replicated as many times as it is needed.[27][28]

The process of clustering is two-fold. The first step is cluster head nomination and then the process of cluster formation begins. In the last ten years many researchers have tried to come up with clustering protocols that provide solutions to the above mentioned unique challenges. 
Most of the known clustering algorithms for WSNs can be further distinguished into two main categories, depending on cluster formation criteria and parameters used for $\mathrm{CH}$ election:

In the category of probabilistic selection clustering algorithms, a priori probability assigned toeach sensor node is used to determine the initial $\mathrm{CHs}$ (or some other type random election procedure is scheduled).[29]. In the category of nonprobabilistic clustering algorithms, more specific (deterministic) criteria for $\mathrm{CH}$ election and cluster formation are primarily considered, which are mainly based on the nodes proximity (connectivity, degree, etc.) and on the information received from other closely located nodes[29]. Cluster based architectures improve the resource allocation and reduce the energy consumption, thus prolong the network lifetime as much as possible [25].

\section{SYSTEM MODEL}

The hybrid WSN consists of a sink, a large number of sensor nodes and a large number of relay nodes. Static sensor nodes are randomly distributed in the sensing field. They monitor the surrounding environment, generate sensed data periodically and transfer it up towards the sink. To reduce energy consumption, they do not relay traffic from other nodes. Relay nodes without sensing and computing capacities are also static. They are responsible for forwarding data from sensor nodes to the sink. The sink with unlimited energy may be static or mobile, depending on the specific scenario. It is responsible for collecting data generated by sensor nodes in the WSN.

\section{ENERGY BALANCING SCHEME}

\subsection{Clustering}

In most wireless sensor network (WSN) applications nowadays the entire network must have the ability to operate unattended in harsh environments in which pure human access and monitoring cannot be easily scheduled or efficiently managed or its even not feasible at all [46]. Sensors are energy constrained and their batteries usually cannot be recharged. Neighboring sensor nodes generally have the data of similar events because they collect events within a specific area. If each node individually transmits the collected data to the sink node, a lot of energy will be wasted to transmit similar data to the sink node. The sensor nodes are organized into a number of clusters in order to avoid such energy wastes. Clusterbased architectures improve the resource allocation and reduce the energy consumption, thus prolong the network lifetime as much as possible [30]. Each cluster is monitored and controlled by a node, called Cluster-Head $(\mathrm{CH})$. These cluster heads communicate directly with the base station (BS). Other nodes send the data, sensed from the environment to these $\mathrm{CHs}$. CHs first aggregate the data from the multiple sensor nodes, and then finally send it directly to the BS as shown in Fig. 3.1. Hence the two phase process of clustering, the two processes being, optimal cluster head selection and cluster formation is one of the pivotal problems in sensor network applications and can drastically affect the networks communication energy dissipation. 


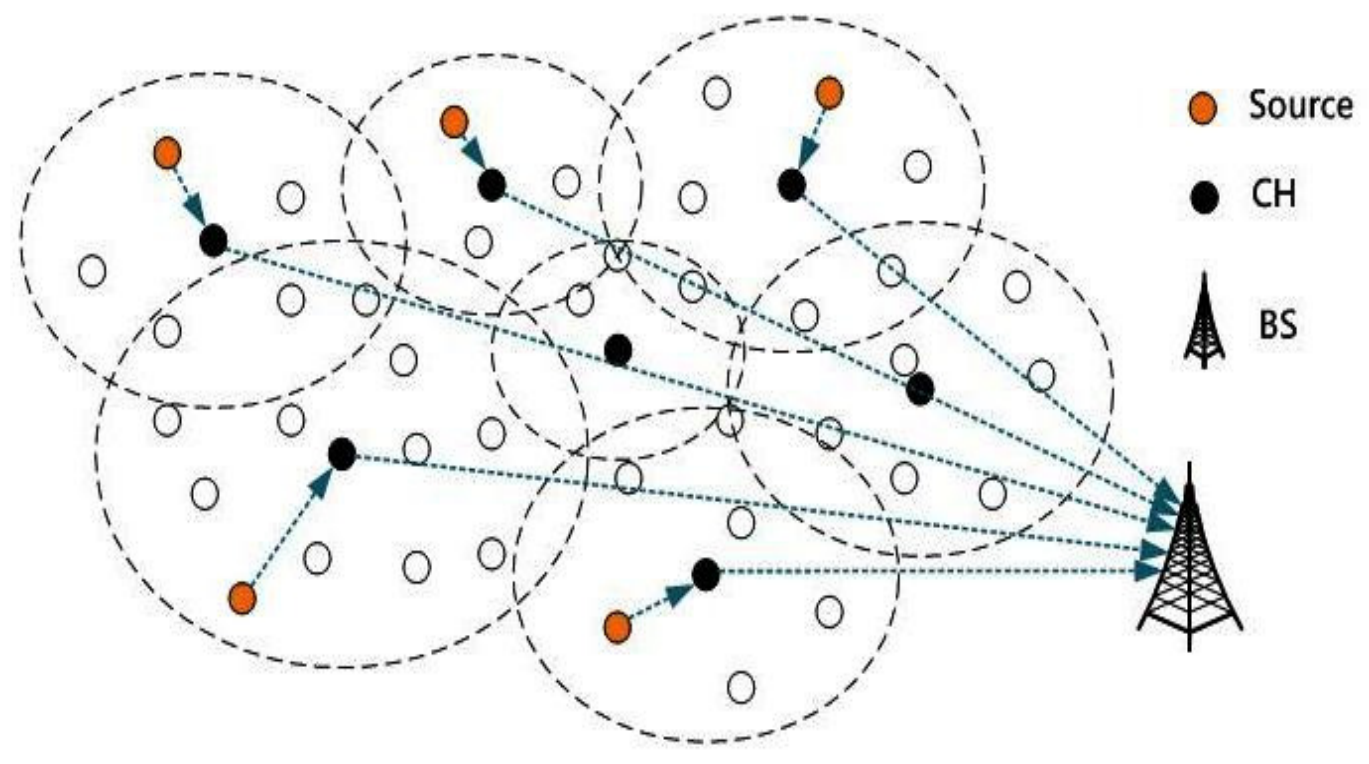

Before documenting on the possible WSNs clustering algorithms, it is worth reporting on some important parameters with regard to the whole clustering procedure in WSNs.

\section{Number of clusters (cluster count)}

In most recent probabilistic and randomized clustering algorithms the $\mathrm{CH}$ election and formation process lead naturally to variable number of clusters. In some published approaches, however, the set of $\mathrm{CHs}$ are predetermined and thus the number of clusters is preset. The number of clusters is usually a critical parameter with regard to the efficiency of the total routing protocol.

\section{Intracluster communication}

In some initial clustering approaches the communication between a sensor and its designated $\mathrm{CH}$ is assumed to be direct. However, multi-hop intracluster communication is often required, i.e., when the communication range of the sensor nodes is limited or the number of sensor nodes is very large and the number of $\mathrm{CHs}$ is bounded.

\section{Nodes and $\mathrm{CH}$ mobility}

If we assume stationary sensor nodes and stationary $\mathrm{CHs}$ we are normally led to stable clusters with facilitated intracluster and intercluster network management. On the contrary, if the $\mathrm{CHs}$ or the nodes themselves are assumed to be mobile, the cluster membership for each node should dynamically change, forcing clusters to evolve over time and probably need to be continuously maintained.

\section{Nodes types and roles}

In some proposed network models the $\mathrm{CHs}$ are assumed to be equipped with significantly more computation and communication resources than others. In most usual network models 
(i.e., homogeneous environments) all nodes have the same capabilities and just a subset of the deployed sensors are designated as CHs.

\section{Cluster-head selection}

The leader nodes of the clusters (CHs) in some proposed algorithms (mainly for heterogeneous environments) can be preassigned. In most cases however (i.e., in homo- geneous environments), the $\mathrm{CHs}$ are picked from the deployed set of nodes either in a probabilistic or completely random way or based on other more specific criteria (residual energy, connectivity etc.).

\section{Algorithm complexity}

In most recent algorithms the fast termination of the executed protocol is one of the primary design goals. Thus, the time complexity or convergence rate of most cluster formation procedures proposed nowadays is constant (or just dependent on the number of $\mathrm{CHs}$ or the number of hops). In some earlier protocols, however, the complexity time has been allowed to depend on the total number of sensors in the network, focusing in other criteria first.

\section{Overlapping}

Several protocols give also high importance on the concept of node overlapping within different clusters (either for better routing efficiency or for faster cluster formation protocol execution or for other reasons). Most of the known protocols, however, still try to have minimum overlap only or do not support overlapping at all.

\subsubsection{LEACH}

LEACH[34] is perhaps the first cluster based routing protocol for WSN, which use a stochastic model for cluster head selection, and has motivated the design of many protocols. Its an hierarchical, probabilistic, distributed, one-hop protocol, with main objectives (a) to improve the lifetime of WSNs by trying to evenly distribute the energy consumption among all the nodes of the network and (b) to reduce the energy consumption in the network nodes (by performing data aggregation and thus reducing the number of communication messages). It forms clusters based on the received signal strength and also uses the $\mathrm{CH}$ nodes as routers to the BS. All the data processing such as data fusion and aggregation are local to the cluster. LEACH forms clusters by using a distributed algorithm, where nodes make autonomous decisions without any centralized control. All nodes have a chance to become $\mathrm{CHs}$ to balance the energy spent per round by each sensor node. Initially a node decides to be a $\mathrm{CH}$ with a probability $\mathrm{p}$ and broadcasts its decision. Specifically, after its election, each $\mathrm{CH}$ broadcasts an advertisement message to the other nodes and each one of the other (non- $\mathrm{CH}$ ) nodes determines a cluster to belong to, by choosing the $\mathrm{CH}$ that can be reached using the least communication energy (based on the signal strength of each $\mathrm{CH}$ message).

The role of being a $\mathrm{CH}$ is rotated periodically among the nodes of the cluster to balance the load.The rotation is performed by getting each node to choose a random number $\mathrm{T}$ between 
0 and 1. A node becomes a $\mathrm{CH}$ for the current rotation round if the number is less than the following threshold:

$$
\mathrm{T}(\mathrm{i})=\frac{\mathrm{p}}{1-\mathrm{p}^{*}(\bmod 1 / \mathrm{p})} \quad \text { if } \mathrm{i} \in \mathrm{G}
$$

else $\mathrm{T}(\mathrm{i})=0$ where

$\mathrm{p}$ is the desired percentage of $\mathrm{CH}$ nodes in the sensor populationr is the current round number

$\mathrm{G}$ is the set of nodes that have not been $\mathrm{CHs}$ in the last 1/p rounds The clusters are formed dynamically in each round and the time to perform the rounds are also selected randomly. Generally, LEACH can provide a quite uniform load distribution in one-hop sensor networks. Moreover, it provides a good balancing of energy consumption by random rotation of CHs. Furthermore, the localized coordination scheme used in LEACH provides better scalability for cluster formation, whereas the better load balancing enhances the network lifetime. However, despite the generally good performance, LEACH has also some clear drawbacks. Because the decision on $\mathrm{CH}$ election and rotation is probabilistic, there is still a good chance that a node with very low energy gets selected as a $\mathrm{CH}$. Due to the same reason, it is possible that the elected $\mathrm{CHs}$ will be concentrated in one part of the network (good $\mathrm{CHs}$ distribution cannot be guaranteed) and some nodes will not have any $\mathrm{CH}$ in their range. Also, the $\mathrm{CHs}$ are assumed to have a long communication range so that the data can reach the $\mathrm{BS}$ directly.This is not always a realistic assumption because the $\mathrm{CHs}$ are usually regular sensors and the BS is often not directly reachable to all nodes. Moreover, LEACH forms in general one-hop intracluster and intercluster topology where each node should transmit directly to the $\mathrm{CHs}$ and thereafter to the $\mathrm{BS}$, thus normally it cannot be used effectively on networks deployed in large regions.

\subsubsection{Energy Efficient ClusterHead selection}

MaChow and Thunder has proposed an energy efficient cluster head(EECH) selection algorithm[29], in which additional parameters have been considered to optimize the process of cluster-head selection. They have achieved energy efficiency in terms of network lifetime, not only in terms of energy consumption. So they extended LEACHs stochastic cluster head selection algorithm by adjusting the threshold $\mathrm{T}(\mathrm{n})$, relative to the nodes remaining energy. Using this threshold each node decides whether or not to become a cluster-head in each round.

$$
T(n)=\left(\frac{p}{l-p(r \bmod l / p)} \frac{E_{\text {residual }}}{E_{\text {initial }}} k_{\text {opt }}\right)
$$

where the $E_{\text {residual }}$ is the remaining energy of the node and $\mathrm{E}_{\text {initial }}$ is the initial energy of the node before the transmission. 


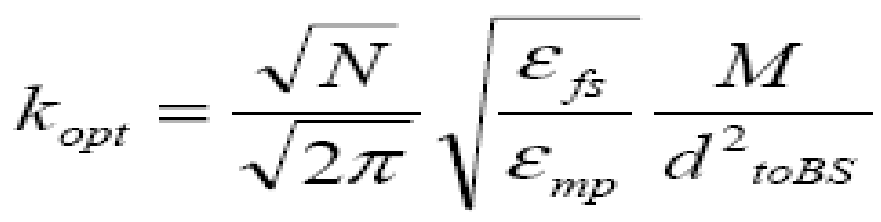

This algorithm is being considered as an optimized version of LEACH. They have considered two additional parameters viz. Residual energy of cluster head and distance between $\mathrm{CH}$ and BS. The major drawback of EECH is that the parameters required for cluster formation and their relative importance required for clustering are not defined.

\subsubsection{Energy Efficient Clustering Scheme}

MaoYe and Chengfa Li had proposed an energy efficient clustering scheme (EECS)[33] . According to EECS, cluster heads are elected with more residual energy through local radio communication. While electing the cluster heads, nodes become CANDIDATE nodes with a probability $\mathrm{T}$ and then broadcast the CDMPETEHEADMSGs within radio range Rcompete to advertise their wills. Each CANDIDATE node checks whether there is a CANDIDATE node with mare residual energy within the radius Rcompete. Once the CANDIDATE node finds a more powerful CANDIDATE node, it will give up the competition without receiving sub sequential CDMPETEHEADMSGs. Otherwise, it will be elected as HEAD in the end.

In cluster formation phase, each HEAD node broadcasts the HEADAD-MSG across the network, while the PLAIN nodes receive all the HEADADJSGs and decide which cluster to join. Most of existed metric for PLAIN nodes to make decisions is the distance metric. For example in [50] or [51], the PLAIN nodes choose the cluster head that require minimum communication according to the received signal strength. However, pursuing efficient energy consumption of the PLAIN nodes only may lead HEAD nodes exhausted quickly during the data transmission phase. The simulation results of EECS shows that it prolongs the network lifetime as much as 135

\subsubsection{Load Based Clustering Scheme(LBCS)}

Shujuan Jin and Keqiu Li proposed a load balanced clustering scheme (LBCS)[34]. To balance the energy load in the cluster, they presented a scheme for selecting an assistant node in the cluster to help the $\mathrm{CH}$ to transmit the aggregated data to the BS. In other words, after the $\mathrm{CH}$ computes the received data, it sends the aggregated data to its assistant node. Then this assistant node is responsible for the data transmission to the BS. During the setup phase, a predetermined fraction of nodes elect themselves as cluster heads as follows. A sensor node generates a random number between 0 and 1 . If this random number is less than a threshold value [36], it will become a cluster head for the current round. As it needs to select a next-hop cluster head according to the distance between the $\mathrm{CH}$ and the $\mathrm{BS}$, and the remaining energy, when each $\mathrm{CH}$ broadcasts its role, it also tells the plain nodes the related energy and distance information. After all the plain nodes receive this advertisement, they can decide which cluster they want to belong and which node they will have to be acted as its next-hop cluster head. This decision is based on the weighted average of the advertisements signal strength and the cluster heads residual energy. In the wireless communication, the residual energy of the $\mathrm{CH}$ is set as the initial energy minus by the dissipative energy Econ 


$$
\text { CHele }=(1-\alpha) \frac{E_{c h}-E_{\min }}{E_{c h} * d_{c h}}+\alpha \frac{d-d_{\min }}{E_{c h} * d_{c h}}
$$

Where, Ech is the residual energy of the $\mathrm{CH}$, which can be obtained directly during our simulation. dch is the distance between the $\mathrm{CH}$ and this plain node, dmin is the minimal one among these distances, and is a weighting coefficient of these two factors. After each plain node receives the $\mathrm{CHs}$ advertisement message, it elects the cluster head node from the $\mathrm{CHs}$ sequence for itself according to (40). The plain node chooses its $\mathrm{CH}$ comprehensively by considering the $\mathrm{CHs}$ remaining energy and the distance away from itself. Although the algorithm performs well, there is still some space to improve the performance. As adopting the multihop transmission mechanism, all the fused data should be sent to base station, so the nodes closer to the base station will receive large amount of data from other clusters to transmit. This certainly will make these nodes consume excessive energy to deal with the transmitted data, which surely leads to some energy holes.

\subsubsection{Distributed Weight Based Energy-Efficient Hierarchical Clustering}

Two more recent weight-based protocols were proposed in [41,42]. In [41] [DistributedWeightBased Energy-Efficient Hierarchical Clustering (DWEHC)] a corresponding distributed algorithm is given, which aims at high energy efficiency by generating balanced cluster sizes and optimizing the intracluster topology. Each sensor calculates its weight after locating the neighboring nodes in its area. The weight is a function of the sensors residual energy and the proximity to the neighbors. In a neighborhood, the node with largest weight would be elected as a $\mathrm{CH}$ and the remaining nodes become members. At this stage the nodes are considered as first-level members because they have a direct link to the $\mathrm{CH}$. A node

progressively adjusts such membership to reach a $\mathrm{CH}$ using the least amount of energy. Basically, a node checks with its non-CH neighbors to find out their minimal cost for reaching a $\mathrm{CH}$. Given the nodes knowledge of the distance to its neighbors, it can assess whether it is better to stay a first-level member or become a second-level one reaching the $\mathrm{CH}$ over a two-hop path. Figure 12.5, illustrates the structure of the intracluster topology. Compared to HEED, the DWEHC algorithm has been shown to generate more well-balanced clusters as well as to achieve significantly lower energy consumption in intracluster and intercluster communication.

\subsubsection{Topology Adaptive Spatial Clustering}

Similarly, in [42] (Topology Adaptive Spatial ClusteringTASC), the authors propose another distributed algorithm that partitions the network into a set of locally isotropic, nonover- lapping clusters without prior knowledge of the number of clusters, cluster size, and node coordinates. This is achieved by deriving a set of weights that include distance, connec- tivity, and density information within the locality of each node. The derived weights form the terrain for holding a coordinated leader election procedure in which each node selects the node closer to the center of mass of its neighborhood to become its leader. Generally, the weight-based clustering protocols have been shown to produce wellbalanced and stable clusters, in a more systematic and deterministic way. Additionally they can achieve better distribution of energy consumption 
because most of them consider the residual energy as part of the computed weights during the $\mathrm{CH}$ election process. However, they normally suffer from the same disadvantages (increased communication time, no $\mathrm{CHs}$ rotation, etc.)

\subsection{Relay node placement}

Various studies with different network configurations and assumptions have been extensively studied. A novel scheme is proposed for optimizing the deployment of the relay nodes within the sensor network[16]. Given the location of the sensors within the network, in order to determine the optimised required relay nodes and their optimal positions in our scheme, it adopts the Voronoi diagram. The contributions of this paper are to balance the traffic load in such a way as to resolve the hotspot problem in the sensors around the nodes, thereby improving the system lifetime, to decide the optimal deployment locations to minimise the total number of relay nodes required and to reduce the transmission delay within the network.

Under the assumption that the relay node has the same dimension and power supply as the sensor node, but is much cheaper because of the lack of sensing and computing capacities[17]. Based on balancing power consumption among all sensor nodes and relay nodes, it deduced a general relay node density function and further extend it to two special cases where the sensing field is a rectangle and the sink is static or mobile. Simulation results show that the energy utilisation is nearly maximised when the number of relay nodes is taken from the proposed density function, and the achieved prolonging of network lifetime is significant compared with uniformly placing relay nodes in both cases where the sink is static and mobile.

When the sensor nodes are prefixed and pre-known [18] can be used. The major contri- bution of this paper is proposal of a two-phase relay node placement strategy with evenly-

consumed energy among different nodes for a more balanced sensor lifetime along with accurate numerical locations and transmission radiuses of relay nodes. It also studies the relationship between the number of relay nodes and major factors such as network lifetime LF, initial energy co, data amount etc., providing an overall reference for the trade-off be- tween the decrease of the number of relay nodes and the prolonged network lifetime with consideration of energy and data amount. For constrained Relay node placement $\mathrm{O}(1)$ approximation algorithms have been formulated in [19]. The single-tiered hetrogenous net- work meets the connectivity and survivability requirements. The constrained study however allows relay nodes to be placed in only a set of candidate locations. For two-tiered archi- tecture with similar constraint of allowing Relay Nodes at only some candidate locations [20] can be used. It studies the connected single-cover problem where each sensor node is covered by a relay node and the relay nodes form a connected network with the base stations. To meet the survivability requirement it studies the 2-connected double-cover problem where each sensor node is covered by at least two relay nodes, and the relay nodes form a 2-connected network with the base stations. For the connected single-cover problem, $\mathrm{O}(1)$ approximation ratios algorithm and for the 2-connected double-cover problem, $\mathrm{O}(1)$ approximation ratios for practical settings and $\mathrm{O}(\mathrm{ln} \mathrm{n})$ approximation ratios for arbitrary settings algorithm is proposed. Energy provisioning (EP) for wireless sensor networks is also important.[21] Considers a two-tier wireless sensor network and studies the joint problem of EP and relay node placement (EPRNP) for the upper tier aggregation and forwarding nodes (AFNs) to increase network lifetime. Since the EPRNP problem formulation is NP-hard,it developed a heuristic algorithm, smart pairing and INtelligent Disc Search (SPINDS), that solves the EPRNP problem. The polynomial running 
time property of SPINDS in this paper was achieved by transforming the original mixed-integer nonlinear programming (MINLP) problem into an iterative LP problem.

QoS parameters like Fault-tolerance have been studied in [22,23]. [22] uses RN as gate- ways and forms clusters for two-tiered WSN. Given a set of sensor nodes in an Euclidean plane, it places minimum number of relay nodes to forward data packets from sensor nodes to the sink, such that:

1. the network is connected

2. the network is 2-connected.

For case one, a $(6+\varepsilon)$-approximation algorithm for any $\varepsilon>0$ with polynomial running time when $\varepsilon$ is fixed is proposed with theoretical proof. And for case two, 2 approximation algorithms with $(24+\varepsilon)$ and $(6 / T+12+\varepsilon)$ respectively, where $T$ is the ratio of the number of relay nodes placed in case one to the number of sensors is proposed. [23] however studies for problems related to fault tolerant. Algorithm for single-tiered fault tolerant relay node placement with and without base sta- tions and algorithm for two-tired WSN without base station is proposed. The algorithm has $\mathrm{O}(1)$ approximation complexity.

\section{CONCLUSION}

Two approaches have been discussed for efficient energy utilization in WSNs viz. clustering and relay node placement. Generally, clustering in WSNs has been of high interest in the last decade and there is already a large number of related published works. Throughout this paper we tried to present the main characteristics of the most significant protocols that were proposed till now in the literature. As it was pointed out, grouping nodes into clusters, thus leading to hierarchical routing and data gathering protocols, has been regarded as the most efficient approach to support scalability in WSNs. The hierarchical cluster structures facilitate the efficient data gathering and aggregation independent to the growth of the WSN, and generally reduce the total amount of communications as well as the energy spent.

Finally, several additional issues should be further studied in future research. Some of the most challenging of these issues include the development of a generic method for finding the optimal number of clusters in order maximize the energy efficiency, the estimation of the optimal frequency of $\mathrm{CH}$ rotation/reelection to gain better energy distribution, however, keeping the total overhead low, the efficient support of nodes and CHs mobility as well as the support of mobile sinks, the incorporation of several security aspects (i.e., enhanced protection needed in hostile environments when cluster-based protocols are used), the further development of efficient recovery protocols in case of $\mathrm{CHs}$ failure, etc

\section{REFERENCES}

[1] I.F. Akyildiz, W. Su, Y. Sankarasubramaniam and E. Cayirc Wireless sensor networks:a survey; COMNET Vol. 38(2002), pp. 393422

[2] J. Bredin, E. Demaine, M. Hajiaghayi and D. Rus Deploying sensor networks with guaranteed capacity and fault tolerance Mobihoc05; pp. 309-319. 
[3] X. Cheng, D.Z. Du, L. Wang and B. Xu; Relay sensor placement in wireless sensor networks; ACM/Springer WINET07

[4] A. Efrat, Sandor P. Fekete, P. Gaddehosur, J. Mitchell, V. Polishchuk and J. Suomela Improved Approximation Algorithms for Relay Placement ESA08; pp.356367.

[5] B. Hao, J. Tang and G. Xue Fault-tolerant relay node placement in wireless sensor networks: formulation and approximation HPSR04; pp. 246-250.

[6] Y.T. Hou, Y. Shi, H.D. Sherali and S.F. Midkiff Prolonging sensor network lifetime with energy provisioning and relay node placement; Secon05; pp. 295304.

[7] A. Kashyap, S. Khuller and M. Shayman Relay placement for higher order connectivity in wireless sensor networks Infocom06.

[8] H. Liu, P.J. Wan and X.H. Jia Fault-tolerant relay node placement in wireless sensor networks; LNCS; Vol. 3595(2005), pp. 230239.

[9] E. Lloyd and G. Xue Relay node placement in wireless sensor networks; IEEE Transac- tions on Computers; Vol. 56(2007), pp. 134138.

[10] Relay node deployment strategies in heterogeneous wireless sensor networks: multiple- hop communication case; Secon05, pp. 575-585.

[11] Yu, C.W., Wei, S.S., Shie, B.J.: Activating the hot spot problem by switching multiple sinks in wireless sensor networks. Proc. Fifth Int. Conf. Mobile Ad-hoc and Sensor, Networks 2009, pp. 204211

[12] Marta, M., Cardei, M Improved sensor network lifetime with multiple mobile sinks, Pervasive Mobile Comput., 2009, 5, (5), pp. 542555

[13] J. Bredin, E. Demaine, M. Hajiaghayi, and D. Rus Deploying sensor networks with guaranteed capacity and fault tolerance, in Proc. ACM MobiHoc, 2005, pp. 309319.

[14] J. Pan, Y. T. Hou, L. Cai, Y. Shi, and S. X. Shen, Topology control for wireless sensor networks, in Proc. ACMMobicom, 2003, pp. 286299.

[15] W. Zhang, G. Xue, and S. Misra, Fault-tolerant relay node placement in wireless sensor networks: Problems and algorithms, in Proc. IEEE INFOCOM, 2007, pp. 16491657.

[16] J.-S. Li H.-C. Kao J.-D. Ke, Voronoi-based relay placement scheme for wireless sensor networks in ietdl 2008

[17] K. Lu G. Liu R. Mao Y. Feng , Relay node placement based on balancing power con- sumption in wireless sensor networks in ietdl 2010

[18] Yue Yao, Yong Fant, and Haiyan Luo ,A Distributed Relay Node Placement Strategy Based on Balanced Network Lifetime for Wireless Sensor Networks IEEE 2010

[19] Satyajayant Misr, Seung Don Hong, Guoliang (Larry) Xue and Jian Tang Constrained Relay Node Placement in Wireless Sensor Networks: Formulation and Approximations in IEEE/ACM 2010

[20] Dejun Yang, Satyajayant Misra, Xi Fang, Guoliang Xue, and Junshan Zhang, Two- Tiered Constrained Relay Node Placement in Wireless Sensor Networks: Efficient Ap- proximations in IEEE 2010

[21] Y. Thomas Hou, Yi Shi, Hanif D. Sherali, and Scott F. Midkiff, On Energy Provisioning and Relay Node Placement for Wireless Sensor Networks in IEEE 2005

[22] Hai Liu, Peng-Jun Wan, and Xiaohua Jia , Fault-Tolerant Relay Node Placement in Wireless Sensor Networks , L. Wang (Ed.): COCOON 2005, LNCS 3595, pp. 230239, 2005. SpringerVerlag Berlin Heidelberg 2005

[23] Weiyi Zhang, Guoliang Xue and Satyajayant Misra Fault-Tolerant Relay Node Place- ment in Wireless Sensor Networks: Problems and Algorithms in IEEE 2007

[24] Jian Tang, Bin Hao, Arunabha Sen, Relay node placement in large scale wireless sensor networks , Science Direct 2006

[25] Do-hyun Nam, hong-ki min, An Efficient Ad-Hoc Routing Using aHybrid Clustering Method in a Wireless Sensor Network, Wirelessand Mobile Computing, Networking and Communications, pp. 60-60, Oct 2007

[26] Ma Chaw Mon Thein, Thandar Thein , An energy efficient Cluster Head Selection for Wireless Sensor network, 2010 Int. Conf. on Intelligent systems, Modelling and simulation.

[27] O.Younis, et. al., HEED: A Hybrid, Energy-Efficient, Distributed Clustering Approach for AdHoc Sensor Networks, IEEE Transactions on Mobile Computing, 3(4):660-669,2004 
[28] S. Lindsey, et. al. ,PEG ASIS: Power-Efficient Gathering in Sensor Information Sys- tems, IEEE Aerospace Conference Proceedings, Vol. 3, 9-16 gust, pp. 102-114, 2002.

[29] Shujuan Jin, Keqiu Li, LBCS: A Load Balanced Clustering Scheme in Wireless Sensor Networks, 2009 Third International Conference on Multimedia and Ubiquitous Engi- neering, IEEE

[30] Suan Khai Chong, Mohamed Medhat Gaber, A rule Learning approach to energy effiecient clustering in wireless sensor network, 2008 IEEE

[31] W.R. Heinzelman, A.P. Chandrakasan, and H. Balakrishnan, Energy efficient commu- nication protocol for wireless microsensor networks, in Proceedings of the 33rd Hawaaian Interantional Conference on System Sciences, January 2000.

[32] W.B. Heinzelman,A.P.Chandrakasan, andH. Balakrishnan, An application specific pro- tocol architecture for wireless microsensor networks, IEEE Transactions on Wireless Communications, 1(4), 660670, 2002.

[33] S. Bandyopadhyay and E. Coyle, An energy efficient hierarchical clustering algorithm for wireless sensor networks, in 22nd Annual Joint Conf. of the IEEE Computer and Communications Societies (INFOCOM 2003), San Francisco, CA, April 2003.

[34] O. Younis and S. Fahmy, HEED: A hybrid, energy-efficient, distributed clustering ap- proach for Ad Hoc sensor networks, IEEE Transactions on Mobile Computing, 3(4), 366379, 2004.

[35] V. Loscri, G. Morabito, and S. Marano, A two-level hierarchy for low-energy adaptive clustering hierarchy, Proceedings of IEEE VTC Conference 2005, Vol. 3, pp. 18091813, 2005.

[36] A. Youssef, M. Younis, M. Youssef, and A. Agrawala, Distributed formation of overlap- ping multihop clusters in wireless sensor networks, in Proceedings of the 49th Annual IEEE Global Communication Conference (Globecom06), San Francisco, CA, Nov. 2006.

[37] S. Selvakennedy and S. Sinnappan, An adaptive data dissemination strategy for wireless sensor networks, International Journal of Distributed Sensor Networks, 3(1), AQ3 2007.

[38] M. Ye, C. Li, G. Chen, and J. Wu, EECS: An energy efficient clustering scheme in wireless sensor networks, in Proceedings of IEEE Intl. Performance Computing and Communications Conference (IPCCC), pp. 535540, 2005.

[39] A. Manjeshwar and D.P. Agrawal,TEEN:Arouting protocol for enhanced efficiency in wireless sensor networks, in Proceedings of the 15th International Parallel and Dis- tributed Processing Symposium, San Francisco, CA, 2001

[40] A. Manjeshwar and D.P. Agrawal, APTEEN: A hybrid protocol for efficient routing and comprehensive information retrieval in wireless sensor networks, in Proceedings of the International Parallel and Distributed Processing Symposium, 2002

[41] S. Yoon and C. Shahabi, Exploiting spatial correlation towards an energy efficient Clustered AGgregation technique (CAG), in IEEE International Conference on Commu- nications, pp. 8298, 2005.

[42] S. Yoon and C. Shahabi,The Clustered AGgregation (CAG) technique leveraging spatial and temporal correlations in wireless sensor networks, ACM Transactions on Sensor Networks, 3(1), March 2007.

[43] Y. Xu and H. Qi, Decentralized reactive clustering for collaborative processing in sen- sor networks, in Proceedings of the 10th International Conference on Parallel and Dis- tributed Systems (ICPADS2004), pp. 5461, July 2004

[44] B. Guo and Z. Li, A dynamic-clustering reactive routing algorithm for wireless sensor networks, Wireless Networks Journal, Springer, ISSN 1572-8196 (Online AQ4 Publica- tion), September 2007. 